\title{
Long-term effects of exercise programs among helicopter pilots with flying related LBP
}

\author{
Knut Andersen ${ }^{\mathrm{a}, *}$, Roald Baardsen ${ }^{\mathrm{b}}$, Ingvild Dalen ${ }^{\mathrm{c}}$ and Jan Petter Larsen ${ }^{\mathrm{d}}$ \\ ${ }^{a}$ Department of Research, Stavanger University Hospital, N-4068 Stavanger, Norway \\ ${ }^{\mathrm{b}}$ Department of Neurosurgery, Stavanger University Hospital, N-4068 Stavanger, Norway \\ ${ }^{\mathrm{c}}$ Department of Research, Section of Biostatistics, Stavanger University Hospital, N-4068 Stavanger, Norway \\ ${ }^{\mathrm{d}}$ Network for Medical Sciences, University of Stavanger, 4036 Stavanger, Norway
}

\begin{abstract}
.
BACKGROUND: Flying related transient Low Back Pain (LBP) among helicopter pilots is considered an occupational distress. OBJECTIVE: To examine if exercise programs can alleviate transient LBP.

METHODS: Sixty-five helicopter pilots (92\% males), all reporting flying related LBP, responded to an epidemiological survey and a long-term follow-up, 44.8 months later, comprising questions regarding transient LBP and number of sick leaves. Data from 37 pilots participating in two exercise programs, A; general for LBP, B; focused for lumbar trunk (LT), included information from clinical examinations and muscular endurance tests of the LT before and after intervention. Twenty-eight pilots did not participate in any intervention.

RESULTS: At long-term follow-up $42 \%$ of the pilots still reported flying related transient LBP. Among participants in program B $26 \%$ had persistent pain, $70 \%$ in program A and $46 \%$ among pilots without intervention. Sick-leave reduction was only observed among participants in program B (30\% to $4 \%)$. Upon re-occurrence of LBP symptoms, half of the pilots in program B again performed exercises to improve their pain.

CONCLUSION: This study indicates that exercise programs focused towards lumbar trunk muscular endurance reduces flying related transient LBP and sick-leave among helicopter pilots. These findings may have implications for the pilots' working conditions.
\end{abstract}

Keywords: Muscular endurance, lumbar trunk, sick leave, transient low back pin

\section{Background}

Low back pain (LBP) among helicopter pilots has been recognized as an occupational distress since the 1960s [1,2]. Two pain patterns have been described; a transient pain pattern related to flying and a more chronic general pattern with characteristics like the LBP experienced by the general population. The transient pain pattern related to flying commences and

\footnotetext{
* Corresponding author: Knut Andersen, Department of Research, Stavanger University Hospital, PB 8100, N-4068 Stavanger, Norway. Tel.: +47 98280934; E-mail: knut.andersen@sus.no.
}

intensifies within one to two hours of flying and is characterized as non-radiating, dull and achy in nature. Usually it is localized in the lumbosacral transitional area and may spread into the buttocks and proximal thighs. It usually disappears within hours after flying [1].

It is suggested that the flying related pain may be a precursor to a more chronic pattern. In addition, flying related LBP may negatively influence flying performance by the pilots $[1,3]$. It is therefore important to better understand why this pain appears and how to prevent it.

Several studies have examined anthropometrical

ISSN 1053-8127/18/\$35.00 (C) 2018 - IOS Press and the authors. All rights reserved

This article is published online with Open Access and distributed under the terms of the Creative Commons Attribution Non-Commercial License (CC BY-NC 4.0). 
factors among the pilots as well as occupational factors as ergonomics of the cockpit and design of the cockpit seat for causality [1]. Anti-vibrational generators have been installed in the aircrafts [4] and studies have explored the working position for pilots in mock-up cockpits with and without vibration [5]. These studies have not been able to establish causality between vibration and the experienced pain. In contrast, prolonged constrained sitting by the pilots with endurance related lumbar trunk muscular fatigue has been found to be an important risk factor for this transient LBP [6].

Also, the pilot's individual ability to withstand the occupational distress related to helicopter flying has been studied [6-8]. This concerns military as well as civilian helicopter operations. The pilots' muscular capabilities must be optimal to endure prolonged constrained sitting and the load of the mandatory body mounted safety gear. Strengthening of lumbar muscular endurance may therefore be necessary to achieve reduced flying related pain and to improve the occupational performance.

In the absence of a causal relationship between external factors such as vibration, ergonomics of the cockpit, seat design and requirements for the use of safety gear in relation to flying related transient LBP, we hypothesize that "internal" factor as improved low back muscular capacity of the individual pilot would reduce the occurrence of flying related transient LBP. The objective of the present study was therefore to examine long-term effects and within-group changes in lumbar trunk muscular endurance and self-reported sick leave following participation in two different training programs in comparison to a group of pilots not participating in any interventions.

\section{Methods}

\subsection{Study design}

This study is a pre-post interventional study among commercial helicopter pilots that were allocated to two different training programs. The applied data is drawn from an initial epidemiological study among the helicopter pilots, findings from a short-term follow-up clinical examination of the pilots and data from a longterm follow-up survey among pilots with transient LBP defined as flying related pain on at least 1 of 3 flights last month. We also included reference data from a group of helicopter pilots with transient LBP that did not participate in any of the interventional programs, but who responded to both the initial survey and the long-term follow-up.

The two training regimens, $\mathrm{A}$ and $\mathrm{B}$, implied 36 training sessions over a 135-day period with short-term follow-up examinations at the end of the intervention. The initial questionnaire-based survey was conducted in February-June 2013 and the long-term follow-up survey after a mean time of 44.8 months (SD 1.0) in ultimo December 2015. The two training regimens were carried out between December 2013 and March 2015. Mean time-span between intervention and longterm follow-up was 26.6 months (SD 3.7). Our primary outcome measure was the proportion of pilots still affected by transient LBP at follow-up in the separate groups and secondary outcome was proportion of self-reported sick-leaves during 2014 and 2015. In addition, we have studied factors that could influence a possible improvement among pilots with training program B. The Western Norway Regional Committee for Medical Research approved the project (REK Vest 2010/2254) and the project was registered at ClinicalTrials.gov Identifier NCT01788111.

\subsection{Subjects and allocation of participants}

The inclusion criteria for this study were commercial helicopter pilots with lumbar pain below lower rib and above gluteal crests with or without pain into lower extremities on at least one of three flights during the last month in the initial epidemiology study. In a survey [3] carried out early 2013, 83 helicopter pilots from the helicopter companies Canadian Holding Company (CHC) and Bristow Norway and located in Stavanger/Sola (SVG) and Bergen/Flesland (BGO) reported transient LBP (see Fig. 1, Flowchart). All the pilots were full-time employed and medically fully licensed to operative flying and as such did not have any health-related exclusion factors.

Based upon the initial survey, pilots satisfying the inclusion criteria were invited to a clinical examination and 62 pilots signed a written consent to participate. After the clinical examination 16 pilots withdrew from participation in the training programs because of time constraints due to foreign domicile, no longer any desire to participate as well as a busy family and/or social life. The remaining 46 pilots were invited to participate in an intervention study comprising two different training programs. The pilots were allocated to the two different training programs according to company affiliation and location. Program A included pilots from $\mathrm{CHC}$ in Stavanger and Bristow Norway in 


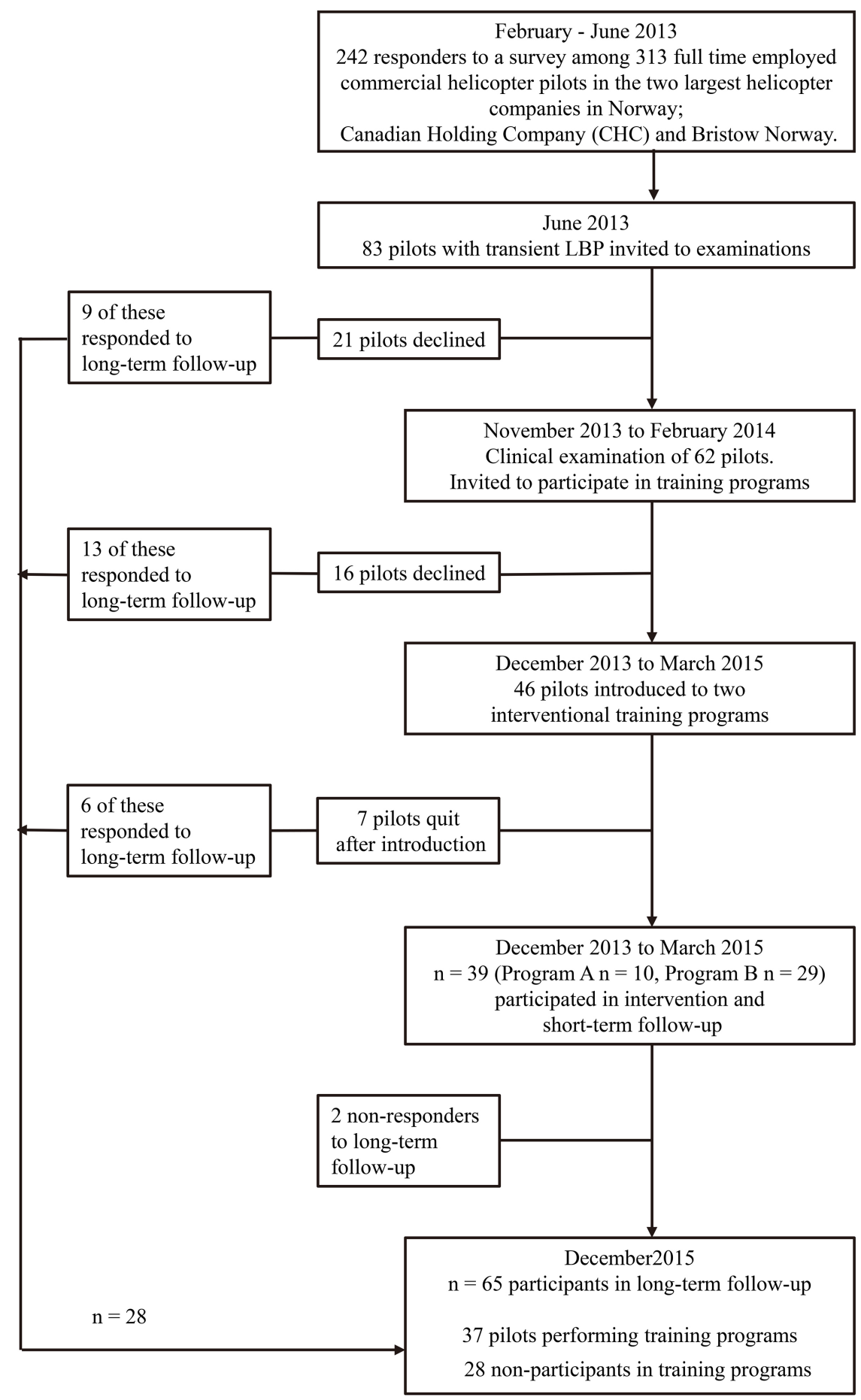

Fig. 1. Flowchart.

Bergen. Conversely, pilots from $\mathrm{CHC}$ in Bergen and Bristow Norway in Stavanger were selected to program B. Directly following the introduction to the exercise programs 7 pilots dropped out declaring no interest in further participation. Due to practical reasons related to small number of participants at the two locations of the two companies respectively, we anticipated difficulties related to compliance when pilots, working and flying 
together, could discuss the two training programs with type and number of exercises as well as differences in execution. Hence, this study did not follow the strict protocol of a randomized control study involving randomization and a control group.

There were 65 responders to the questionnaire based follow-up study conducted in December 2015 among the 83 pilots initially reporting transient LBP. The interventional study had 39 pilots participating with clinical examinations before and 3 months after training. However, only 37 of these responded to the long-term follow-up-survey with 10 pilots from program A and 29 from program B. The total study group thus consisted of 65 pilots, 28 without and 37 pilots with training.

\subsection{Examinations}

In this report, we have used three sources of data as the basis for our analyses. First, data from a survey conducted in 2013. Secondly, data from the clinical examinations before and after intervention and thirdly, data from a long-term follow-up.

The initial survey explored the pilots' age, height, weight and the pilots flying experience in years, total hours and hours last year, number of hours flying "large" helicopters (carrying up to 19 passengers) and type of aircraft operated. The survey also contained questions about LBP on at least one of three flights last month.

The clinical examinations among pilots that reported transient LBP were performed before and after intervention and included relevant orthopedic and neurological tests of the lumbar spine. They scored the intensity of the general LBP last week on a visual analog scale (VAS 0-10) with higher values indicating more intense pain. Level of function of daily living was scored by the Oswestry Disability Index (ODI 0100 ) with higher values indicating poorer function, and quality of life was scored by Euroqol 5D index (EQ5D) ranging from $0-100$ with 0 equal death and with 100 subjectively best possible quality of health. An individual interview with the pilots also investigated aggravating and alleviating factors concerning the LBP with timing factors for pain onset, exacerbation and relief both during or after flying and within hours or days.

The pilots' lumbar trunk muscular endurance was tested without tactile or verbal feedback during the test [9] (see Appendix 1), and measured in seconds in 4 positions; extension, flexion and right and left side bridge. The back extensors were tested in a Biering-
Sorensen position with the upper body cantilevered over the edge of the test bench with secured ankles under the foot support. For the flexion test we applied a 55 degrees angled jig with the feet anchored under the foot support, knees at 90 degrees and the upper torso resting towards the jig. The test started when the jig was pulled back $10 \mathrm{~cm}$. The side bridge tests were performed on either side with elbow support, legs fully extended with the top foot in front of the lower and the body in a straight line. Before starting each test, identical verbal and visual instructions were given to all participants. Similar testing procedure has been validated through reliability studies [10]. The tests were conducted at random by a physiotherapist or the main author after procedural agreement.

The long-term follow-up survey during late December 2015 recorded if the pilots still had flying related transient LBP on at least one of three flights last month. It also comprised questions related to age, sex, location and working status and questions related to flying experience such as type of aircraft operated and total and annual hours and, for each year, questions regarding absence or sick leave due to LBP for 2013 (before intervention), 2014 (the year of intervention) and 2015 (after intervention). Participants in the intervention programs were also asked in general about experienced effect and benefit of the training programs along with questions about how often they had done the exercise programs, beyond the intervention period, up to the time of the long-term follow-up.

\subsection{Interventions}

For the intervention, the pilots were allocated to two training programs per company and location. This was to avoid discussions of efficacy and preference among pilots serving at same base and in same company and possibly flying together. We included both a traditional LBP program A (Appendix 2A) and a program B (Appendix 2B) designed to increase lumbar trunk (LT) muscular endurance. Program A consisted of 10 exercises each performed in series of $3 \times 10$ repetitions. The exercises used in Program A are generally accepted as addressing low back complaints. All pilots allocated to program A had an instruction class led by a physiotherapist with demonstration of the exercises. There were no specific instructions for the execution of the exercises.

Program B consisted of 4 exercises. The exercises were explained and demonstrated either on an individual level or in small groups (maximum 3 pilots) led by 
Table 1

Demographic and clinical data at inclusion of the study for helicopter pilots reporting transient LBP according to intervention or not and according to type of intervention. Results given as mean with standard deviation (SD) unless otherwise stated

\begin{tabular}{|c|c|c|c|c|c|}
\hline & $\begin{array}{c}\text { All pilots } \\
\text { first survey } n=65\end{array}$ & $\begin{array}{c}\text { Pilots without } \\
\text { intervention } n=28\end{array}$ & $\begin{array}{c}\text { Pilots with } \\
\text { intervention } n=37\end{array}$ & $\begin{array}{c}\text { Program A } \\
n=10\end{array}$ & $\begin{array}{c}\text { Program B } \\
n=27\end{array}$ \\
\hline$n$ (\% males) & $60(92 \%)$ & $26(93 \%)$ & $34(91 \%)$ & $9(90 \%)$ & $25(93 \%)$ \\
\hline Age (years) & $38.9(7.4)$ & $40.3(8.6)$ & $38.2(6.8)$ & $38.3(8.8)$ & $38.2(6.1)$ \\
\hline Height $(\mathrm{cm})$ & $180.8(7.4)$ & $180.1(6.5)$ & $181.2(7.9)$ & $182.5(11.8)$ & $180.7(6.1)$ \\
\hline Weight (kg) & $83.8(10.2)$ & $83.3(10.9)$ & $84.0(10.0)$ & $85.5(6.3)$ & $83.4(11.1)$ \\
\hline BMI & $25.4(2.5)$ & $25.0(2.7)$ & $25.6(2.4)$ & $25.7(2.7)$ & $25.5(2.3)$ \\
\hline \multicolumn{6}{|l|}{ Flying experience } \\
\hline Years & $14.9(7.4)$ & $16.9(8.5)$ & $13.3(6.2)$ & $13.6(7.4)$ & $13.2(5.8)$ \\
\hline Total hours & $6040(3640)$ & $6860(4260)$ & $5420(2990)$ & $6070(3890)$ & $5180(2640)$ \\
\hline Hours in large helicopter & $4180(3180)$ & $5010(4020)$ & $3550(2230)$ & $4310(3210)$ & $3270(1730)$ \\
\hline Flying hours last years & $519(183)$ & $480(171)$ & $546(190)$ & $431(168)$ & $589(183)$ \\
\hline \multicolumn{6}{|l|}{ Aircraft } \\
\hline S92 & $47(72 \%)$ & $17(61 \%)$ & $30(81 \%)$ & $3(30 \%)$ & $27(100 \%)$ \\
\hline AS332 L/L2 & $13(20 \%)$ & $6(21 \%)$ & $7(19 \%)$ & $7(70 \%)$ & - \\
\hline $\mathrm{EC} 225$ & $5(8 \%)$ & $5(18 \%)$ & - & - & - \\
\hline \multicolumn{6}{|l|}{ Clinical data } \\
\hline VAS (0-10) LBP & $3.4(2.0)^{1}$ & $3.0(2.0)^{2}$ & $3.6(2.0)$ & $3.0(1.8)$ & $3.8(2.1)$ \\
\hline ODI in percent & $10.7(6.0)^{1}$ & $10.8(5.6)^{2}$ & $10.6(6.3)$ & $8.8(4.8)$ & $11.3(6.9)$ \\
\hline EQ-5D (0-100) & $83.3(12.6)^{1}$ & $84.2(11.7)^{2}$ & $82.8(13.2)$ & $81.8(17.3)$ & $83.2(11.7)$ \\
\hline
\end{tabular}

S92: Helicopter type Sikorsky S92. AS332L/L2; Helicopter type Eurocopter (Aerospatial) models 332 L, L 1 and L 2 . EC225; Helicopter type Eurocopter model $225 .{ }^{1}$ Data from 55 pilots. ${ }^{2}$ Data from 19 pilots.

the main author. Three exercises were performed on an "angle training bench" and one "curl-up" exercise on a floor mat. All pilots were instructed and rehearsed in "bracing" [9]. "Bracing" should be initiated and released between each repetition. Every repetition comprised an initial steady fast concentric phase, followed by an isometric phase and a closing eccentric phase. All exercises were performed in 4 series according to a "reversed pyramid" protocol [9]. In general, the series were separated by one-minute intervals involving brisk walking or cycling on a stationary spinning bicycle. All exercises were performed according to the principle of progressive overload [11].

Following the scheduled 135 days' intervention period we emphasized to the pilots the importance of maintaining the training instructed in the programs [11] and we encouraged the pilots to repeat the training programs when and if exposed to symptoms of transient LBP during follow-up for evaluation of possible long-term effects.

\subsection{Statistical analysis}

Descriptive statistics are given as means and standard deviation (SD) for continuous data and as counts and percentage for categorical data. Characteristics among those with vs without persistent pain in longterm follow-up were compared using independent samples t-tests. McNemar's test was applied to compare number of pilots with sick leaves before, during or after the intervention period. All statistical analysis was performed using IBM SPP Statistics v.23. Statistical significance was defined as $p<0.05$.

\section{Results}

This study included 65 full time employed commercial helicopter pilots. All pilots reported, at inclusion, flying related LBP between the lower rib and gluteal folds on at least 1 of 3 flights last month (transient LBP). Table 1 shows demographic and clinical findings from the initial survey for all pilots as well as data from the short-term clinical examinations. In addition, the table shows data for pilots allocated to the two training programs; A and B.

At clinical examination, the pilots reported their level of general LBP as 3.4 on a VAS (SD 2.0, range 0-10) and a mean ODI score of 10.7 (SD 6.0, range 0100). Quality of health was measured by EQ-5D with a score of 83.3 (SD 12.6, range 0-100). These values show that the pilots had low to moderate low back problems in everyday life.

\subsection{Long-term persistence of transient LBP}

At the long-term follow-up in late December 2015, 27 out of 65 pilots (42\%) still reported transient LBP. Among pilots not participating in any training programs 13 out of 28 pilots $(46 \%)$ reported persisting 
Table 2

McNemar's test comparing sick leave 2014 and 2015 to sick leave 2013 and number and percent of pilots reporting sick-leaves due to LBP for 2013,2014 and 2015 as reported in the long-term follow-up survey

\begin{tabular}{|c|c|c|c|c|c|c|c|c|c|}
\hline & & $\begin{array}{c}\text { No intervention } \\
n=28^{1}\end{array}$ & $\begin{array}{c}p \text { - } \\
\text { value }\end{array}$ & $\begin{array}{c}\text { Program } \mathrm{A}^{2}+\mathrm{B}^{3} \\
n=37\end{array}$ & $\begin{array}{c}p \text { - } \\
\text { value }\end{array}$ & $\begin{array}{c}\text { Program A } \\
n=10\end{array}$ & $\begin{array}{c}p \text { - } \\
\text { value }\end{array}$ & $\begin{array}{c}\text { Program B } \\
n=27\end{array}$ & $\begin{array}{c}p- \\
\text { value }\end{array}$ \\
\hline $\begin{array}{l}\text { Before } \\
\quad \text { intervention }\end{array}$ & Sick leave 2013 & $5(20 \%)$ & & $8(22 \%)$ & & - & & $8(30 \%)$ & \\
\hline $\begin{array}{l}\text { During } \\
\quad \text { intervention }\end{array}$ & Sick leave 2014 & $4(16 \%)$ & 0.655 & $8(22 \%)$ & 1.000 & $1(10 \%)$ & 1.000 & $7(26 \%)$ & 1.000 \\
\hline $\begin{array}{l}\text { After } \\
\quad \text { intervention }\end{array}$ & Sick leave 2015 & $2(8 \%)$ & 0.257 & $2(5 \%)$ & 0.109 & $1(10 \%)$ & 1.000 & $1(4 \%)$ & 0.039 \\
\hline
\end{tabular}

${ }^{1}$ Data from 25 pilots. ${ }^{2}$ Program A; general exercises for LBP. ${ }^{3}$ Program B; focused lumbar trunk exercises for LBP.

transient LBP. Similarly, $14(38 \%)$ of the 37 participants in the intervention programs reported transient LBP. However, among the pilots in program B, only 7 out of 27 pilots (26\%) had persistent transient LBP. Among participants in program A, 7 out of $10(70 \%)$ had persistent LBP.

Numbers of sick-leaves were reported by the pilots for the years 2013 (before intervention), 2014 (the year of intervention) and 2015 (after intervention) in the long-term follow-up study. Table 2 shows no reduction in number of sick-leaves from 2013 to 2014. However, a reduction in sick leave was observed for the pilots from 2013 to 2015 and most strongly among participants in training program B (from $30 \%$ to $4 \%, p=$ 0.039).

During the intervention period, pilots in program A performed a total of 265 training sessions with a mean number of 26.5 (SD 10.4) sessions and participants in program B performed a total of 402 sessions with a mean of 14.9 (SD 10.6). Among the subsets of participants in Program B still reporting transient LBP at the long-term follow-up, 26.6 months (SD 3.7) after the intervention period, the scores were VAS 2.3 (SD 1.0), ODI 7.7 (SD 5.2) and EQ-5D 85.7 (SD 14.0) versus VAS 1.8 (SD 1.5), ODI 6.9 (SD 7.1) and EQ-5D 91.8 (SD 11.9) among the pilots now without transient LBP.

\subsection{Factors influencing long term outcome}

Comparing pilots reporting persistent pain at longterm follow-up versus pilots that did not have persistent pain, we found statistically significant difference in health-related quality of life before intervention, mean 74.4 (SD 4.8) vs. 86.3 (SD 11.8) $(p=$ $0.001)$. The mean difference in score was $11.9(95 \%$ CI: 5.3 to 18.5). There were no similar significant findings for general LBP last week before the intervention as measured by VAS, or function as measured by ODI at inclusion among the pilots with or without persistent pain. After intervention, all pilots reported a mean
VAS score of 2.2 (SD 1.5), an ODI of 6.9 (SD 6.0) and EQ-5D of 89.9 (SD 12.2). Neither were there any significant differences between the pilots in flying experience in years, total hours, hours in large helicopter or flying hours last year. Nor did we find significant differences in age, height, weight or in Body Mass Index. All pilots operated the same aircraft (S92).

In contrast, we found that pilots without persisting transient LBP at long-term follow-up had significantly better improvement in muscular endurance during the intervention than those who still had persisting transient LBP. This was as measured with a total of 4 positions of the lumbar trunk ( $p=0.047)$.

About 2/3 of all the participating pilots found the instructed programs beneficial and they rated the effect from good to very good. Half of the participants in program $\mathrm{B}$ returned to the program if experiencing re-occurring symptoms after the intervention period. On average, they reported needing 3.5 (SD 1.9) training sessions before the symptoms improved. Among the pilots who controlled their symptoms in this manner during the post-interventional period until December 2015 (26.6 months), as many as $84 \%$ reported to having done this on one to four occasions.

\section{Discussion}

This study shows that commercial helicopter pilots with flying related LBP may benefit from performing an exercise program designed to strengthen lumbar trunk muscular endurance. Also, number of sick leaves caused by LBP was reduced in a 2-year followup study of this group of pilots with such complaints. Pilots with traditional LBP exercises did not have the same improvement. Our results indicate that helicopter pilots should be offered focused training programs to reduce these complaints that may impact the pilots' flying performance. Furthermore, the findings are in line with the assumption that normal functioning of the 
lumbar trunk muscles is important to avoid or reduce LBP.

Commercial helicopter pilots have a demanding job situation that requires optimal vigilance and performance when transporting oil-workers to and from the oil platforms in the North Sea. Any pain or discomfort may influence their work negatively. The longknown LBP related to flying that these pilots experience may have such a negative influence on performance. A review indicates this is the case among 30\%$88 \%$ of both civilian and military helicopter pilots [1]. It has therefore been important to find ways to decrease the pilots' LBP when flying and the major focus has been on improving the physical working environment for the pilot. Both companies involved in this study have installed anti-vibrational generators in their large helicopters and the helicopters are equipped with the best cockpit seats available. Still the pilots are applying elastic foam pillows either under the buttocks and or in the lumbar area to compensate for the perceived lack of necessary upholstery in the seats. The mandatory body mounted safety gear also contributes by its weight and volume to the constrained ergonomically working conditions in the cockpit.

An alternative strategy to prevent this LBP is by developing ways to increase the pilots' resistance to the pain. Based on data showing that transient LBP is provoked by awkward sitting and followed by decreased lumbar trunk muscular function, it has been postulated that training of these muscles could reduce the pain $[6,7]$. In a short-term study, we showed that exercise programs could improve pain, function and quality of health and this was related to a marked improvement of muscular endurance of the lumbar trunk [12]. In the present study, we have examined the persistence of flying related LBP at mean 26.6 months after intervention. We found that pilots who had trained the program designed to strengthen the muscular trunk had considerably less flying related LBP and less sick leave caused by such pain. Only $26 \%$ of them still had transient LBP. In the group of pilots doing traditional LBP exercises $70 \%$ still experienced LBP when flying. A third group of pilots had not been instructed in any exercises. They had less important complaints initially and about half of them had spontaneously improved. This shows that these complaints have a natural variation over time. Still, the marked improvement of pilots who had a training program to specifically strengthen the lumbar trunk indicates that our findings are valid and important. These findings are in line with data from a randomized controlled study among US
Air Force helicopter pilots, also with few participants, resulting in decreased in-flight pain and disability following a core strengthening exercise program [7].

Several studies claim that the primary causal factor for flight related pain is posture and muscular fatigue due to prolonged periods of static positioning during the flight $[1,2,13]$. It is hypothesized that this will lead to lumbar extensor deconditioning $[14,15]$ and the importance of strengthening the lumbar trunk muscles is supported by the fact that the pilots who in this study did not become pain free, had much less improvement of lumbar trunk endurance from pre-to post intervention. Furthermore, a substantial proportion of the pilots reported that when experiencing recurrence of flying related pain, they returned to the instructed exercises and thereby improved their symptoms.

These observations may have practical consequences for how a helicopter pilot should prepare their muscular defense against LBP in their job situation. In addition, these findings strongly support that normal functioning of the lumbar trunk muscles is important to avoid or reduce LBP in general. These findings are in line with a systematic review and metaanalysis on prevention of LBP [16] that suggests that exercises alone or in combination with education is effective for preventing LBP. The meta-analysis encompassed, among others, studies of core exercises emphasizing the strengthening of back and abdominal muscles. These studies reported an effect size of reduction in risk for subsequent LBP between $25 \%$ to $40 \%$ with some evidence of reduced use of sick leave. The review, however, states that many of the evaluated studies had no effect after 1 year. They explain this by reduced adherence to the exercise programs. The present study showed that the pilots without pain maintained their training throughout the long-term follow-up period.

The practical implication of this study for commercial helicopter pilots is to be aware of the LBP they may experience during their working career, and also, that they themselves and their companies should facilitate and implement systematic programs, and adhere to them $[17,18]$ in order to strengthen lumbar trunk muscles to avoid transient LBP and thereby optimize flying performance.

An important limitation of this study is the lack of a formal randomized controlled design. Also, the small number of participating pilots limits the statistical power to show differences or correlations. Still, we have supporting results that indicates that our main findings are valid and important. We have also in this 
study data from several sources and a rather long follow-up period. In addition, we recognize the limitations related to lack of blinding, as well as high attrition among the participants. In future studies, it is necessary to conduct better controlled studies with larger populations to further investigate this important issue related to both practical advice for commercial helicopter pilots and the understanding of the lumbar trunk muscles and LBP.

\section{Conclusion}

This study indicates that an exercise program focused towards lumbar trunk muscular endurance reduces flying related transient LBP among commercial helicopter pilots. It also indicates that number of sick leaves due to flying related LBP may be reduced among pilots performing such a training program either regularly or at intervals. Better designed and larger studies are needed to investigate this topic further.

\section{Acknowledgments}

Study financed by the Working Environment Fund of NHO; The Confederation of Norwegian Enterprise along with NHO; Federation of Norwegian Aviation Industries (NHO Luftfart) and the Research foundation ELIB (Et Liv I Bevegelse). Also, our appreciation to the two helicopter companies; Canadian Holding Company $(\mathrm{CHC})$ and Bristow Norway and their respective locations in Stavanger and Bergen.

\section{Conflict of interest}

None to report.

\section{References}

[1] Gaydos SJ. Low back pain: considerations for rotary-wing aircrew. Aviat Space Environ Med. 2012; 83(9): 879-89.

[2] Lis AM, Black KM, Korn H, Nordin M. Association between sitting and occupational LBP. Eur Spine J. 2007; 16(2): 28398. doi: 101007/s00586-006-0143-7.

[3] Andersen K, Baardsen R, Dalen I, Larsen JP. Recurrent and Transient Spinal Pain Among Commercial Helicopter Pilots. Aerosp Med Hum Perform. 2015; 86(11): 962-9. doi: 103357/AMHP.4237.2015.

[4] Kasin JI, Mansfield N, Wagstaff A. Whole body vibration in helicopters: risk assessment in relation to low back pain. Aviat Space Environ Med. 2011; 82(8): 790-6.
[5] Shanahan DF, Reading TE. Helicopter pilot back pain: a preliminary study. Aviat Space Environ Med. 1984; 55(2): 117 21.

[6] Pelham TW, White H, Holt LE, Lee SW. The etiology of low back pain in military helicopter aviators: prevention and treatment. Work. 2005; 24(2): 101-10.

[7] Brandt Y, Currier L, Plante TW, Schubert Kabban CM, Tvaryanas AP. A Randomized Controlled Trial of Core Strengthening Exercises in Helicopter Crewmembers with Low Back Pain. Aerospace Medicine and Human Performance. 2015; 86(10): 889-94. doi: 103357/AMHP.4245.2015.

[8] Rochelt HS. Trunk muscle endurance of military pilots: preliminary results of a standardized testing and training program. Aviation, Space and Environmental Medicine. 2010; 81(3): 277.

[9] McGill S. Low back disorders: evidence-based prevention and rehabilitation: Human Kinetics; 2007.

[10] Waldhelm A, Li L. Endurance tests are the most reliable core stability related measurements. Journal of Sport and Health Science. 2012; 1(2): 121-8.

[11] American College of Sports Medicine position stand. Progression models in resistance training for healthy adults. Medicine and Science in Sports and Exercise. 2009; 41(3): 687-708. doi: 101249/MSS.0b013e3181915670.

[12] Andersen K, Baardsen R, Dalen I, Larsen JP. Impact of exercise programs among helicopter pilots with transient LBP. BMC Musculoskeletal Disorders. 2017; 18(1): 269. doi: 10.1186/s12891-017-1631-0.

[13] Balasubramanian V, Dutt A, Rai S. Analysis of muscle fatigue in helicopter pilots. Appl Ergon. 2011; 42(6): 913-8. doi: 101016/j.apergo.2011.02.008.

[14] Steele J, Bruce-Low S, Smith D. A reappraisal of the deconditioning hypothesis in low back pain: Review of evidence from a triumvirate of research methods on specific lumbar extensor deconditioning. Current Medical Research and Opinion. 2014; 30(5): 865-911. doi: 101185/03007995.2013.875465.

[15] Todd AI, Bennett AI, Christie CJ. Physical implications of prolonged sitting in a confined posture-A literature review. Ergonomics SA. 2007; 19(2): 7-21.

[16] Steffens D, Maher CG, Pereira LS, Stevens ML, Oliveira VC, Chapple M, et al. Prevention of low back pain: a systematic review and meta-analysis. JAMA Internal Medicine. 2016; 176(2): 199-208.

[17] Carey TS, Freburger JK. Exercise and the prevention of low back pain: Ready for implementation. JAMA Internal Medicine. 2016; 176(2): 208-9.

[18] Sjøgaard G, Christensen JR, Justesen JB, Murray M, Dalager $\mathrm{T}$, Fredslund GH, et al. Exercise is more than medicine: The working age population's well-being and productivity. Journal of Sport and Health Science. 2016.

\section{Appendix 1}

Testing procedure for lumbar trunk muscular endurance [9]

Equipment: Angle bench, floor mat, $55^{\circ}$ angled jig (equipped with anti-skid material on the bottom) and stop watch

General Instructions: This is to measure in seconds your lumbar trunk muscular endurance in 4 posi- 
tions; extension, flexion and side-bridge on either side. The purpose is to measure how long you can maintain a steady posture in each position and the time starts once you are guided into the correct position and you say a clear and loud YES. You can stop whenever you like. You will not receive any verbal or tactile feedback during the test. If you deviate from the assigned position I will stop the test. You will be allowed some movement. (Intertester agreement allows $5 \mathrm{~cm}$ ). There will be at least a 3-minute rest between the different tests.

\section{Test 1 Extension}

Bench settings: Horizontal foot piece. Chest piece lowered to $60^{\circ}$ to give some support during positioning only.

The candidate is placed prone on the angle bench with feet securely fastened under the foot support. The body is placed with anterior superior iliac crest cantilevered on the superior edge of the table's foot piece, Hands and arms crossed on opposite shoulder in front of the chest. Candidate lifts the upper torso and is guided to a straight body line.

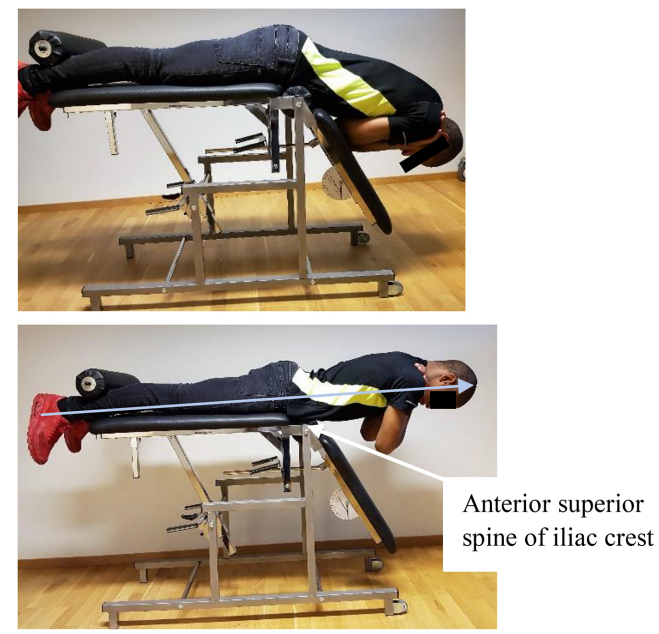

Test 2 flexion

Bench settings: The entire bench is horizontal. The jig is put on top and position is adjusted to the length of the individual test person.

The candidate sits on top of the bench with feet firmly secured under the foot support with a knee angle of $90^{\circ}$. The upper torso is leaned against the $55^{\circ}$ angled jig. The candidate is instructed to maintain that $55^{\circ}$ angle according to general instructions as outlined above. The starts when the jig is pulled back $10 \mathrm{~cm}$.
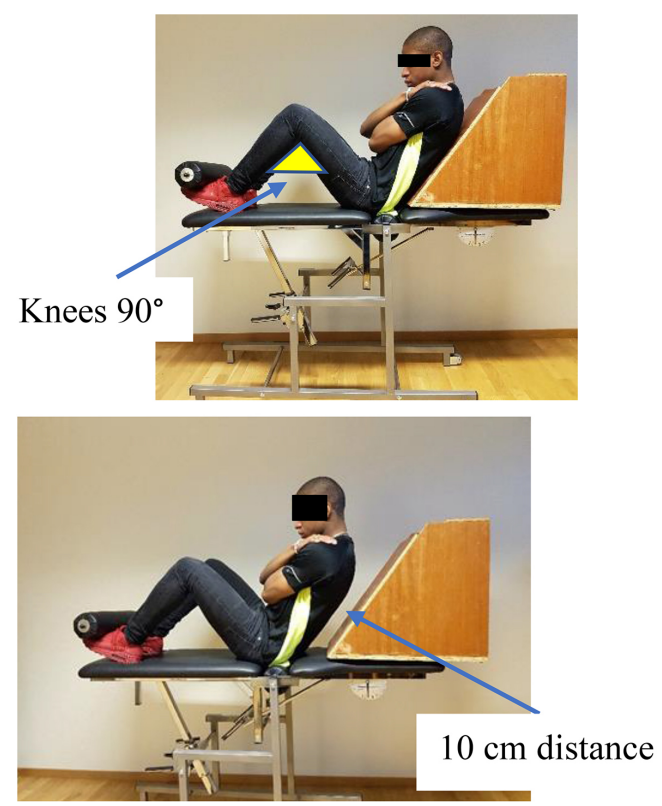

Test 3 Side bridge on either side

Right side up first as a procedure.

\section{Floor mat.}

The candidate is placed sideways on the mat with straight knees and upper leg in front of the lower with toe against heel. Upper body is supported with elbow on the floor resting free hand supporting the weightbearing opposite shoulder. The test starts once the body is in a straight line.

McGill S. Low back disorders: evidence-based prevention and rehabilitation: Human Kinetics; 2007.

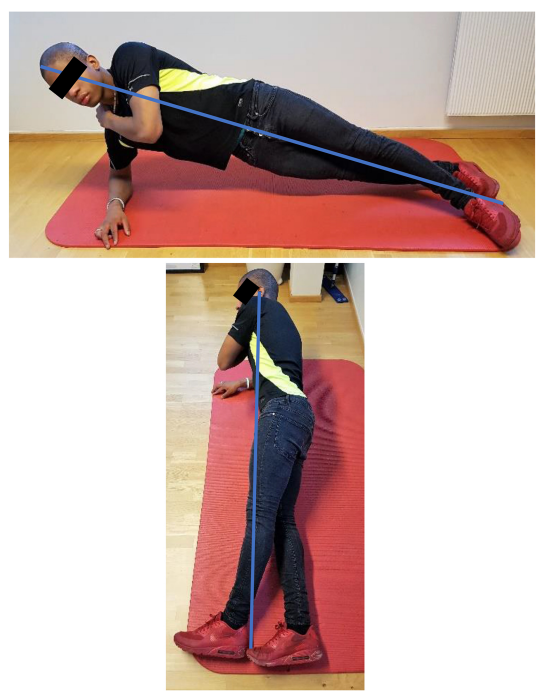




\section{Appendix 2A}

Workout plan Program A

Helicopter pilots with transient low back pain.

All exercises: 3 series $\times 10$ repetitions EXORLIVE

\begin{tabular}{|c|c|c|c|c|c|c|}
\hline Exercise & Illustration & & Focus & & Exercise data & Comments \\
\hline $\begin{array}{l}1 \text { - } \\
\text { Arching swaying, } \\
\text { standing on all } \\
\text { fours }\end{array}$ & & & 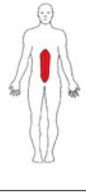 & 38 & $3 \times 10$ & $\begin{array}{l}\text { Start on all fours. Arch your back, } \\
\text { looking down towards your navel } \\
\text { and hold the position for } 4-5 \mathrm{sec} \text {. } \\
\text { Reverse the arch looking up and } \\
\text { hold for } 4-5 \mathrm{sec} \text {., then rest for } 4 \text { - } \\
5 \mathrm{sec} \text {. }\end{array}$ \\
\hline $\begin{array}{l}2 \text { - } \\
\text { "Bridge" }\end{array}$ & & & & & $3 \times 10$ & $\begin{array}{l}\text { Lie on your back with the soles } \\
\text { of your feet on the floor and } \\
\text { your arms out to the side. Tilt } \\
\text { your pelvis backward. Activate } \\
\text { the lower part of your } \\
\text { abdominal muscles, pull your } \\
\text { navel inward and "flatten" your } \\
\text { abdomen. Raise your pelvis } \\
\text { from the floor until you lie on } \\
\text { your shoulder blades only. } \\
\text { Lower and raise slowly }\end{array}$ \\
\hline $\begin{array}{l}3 \text { - } \\
\text { Diagonal lift, } \\
\text { standing on } \\
\text { all fours }\end{array}$ & & & 4 & & $3 \times 10$ & $\begin{array}{l}\text { Start on all fours with your face } \\
\text { facing the floor. Contract your } \\
\text { abdomen and lower back for } \\
\text { stability. Stretch one arm and the } \\
\text { opposite leg to extend of your } \\
\text { body. Repeat the movement with } \\
\text { opposite arm and leg. }\end{array}$ \\
\hline $\begin{array}{l}4- \\
\text { Lying pelvis } \\
\text { rotation } 2\end{array}$ & 5 & & $\int$ & & $3 \times 10$ & $\begin{array}{l}\text { Lie on your back with your hips } \\
\text { and knees bent } 90 \text { degrees and } \\
\text { arms lying to the sides. } \\
\text { Alternately rotate your pelvis } \\
\text { and legs to your right and left. } \\
\text { Keep your legs together. }\end{array}$ \\
\hline $\begin{array}{l}5 \text { - } \\
\text { Diagonal lift, } \\
\text { lying face down }\end{array}$ & & & $\int 8$ & & $3 \times 10$ & $\begin{array}{l}\text { Lie flat, facing down with your } \\
\text { arms straight above your head. } \\
\text { Lift one arm and opposite leg, } \\
\text { in stretched position, off the } \\
\text { floor and slowly lower them } \\
\text { again. Lift opposite arm and leg. }\end{array}$ \\
\hline $\begin{array}{l}6 \text { - } \\
\text { Squat stability }\end{array}$ & $\infty$ & & 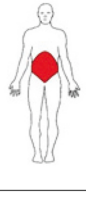 & & $3 \times 10$ & $\begin{array}{l}\text { Stand erect and find the } \\
\text { neutral position of your back } \\
\text { and pelvis. "Flatten" your } \\
\text { abdomen and bend your knees } \\
\text { until in a seated position. Push } \\
\text { back up and repeat. }\end{array}$ \\
\hline $\begin{array}{l}7- \\
\text { Pelvis lift } \\
\text { w/knee } \\
\text { stretching }\end{array}$ & & & & Ul & $3 \times 10$ & $\begin{array}{l}\text { Lie on your back with bent knees } \\
\text { and your feet on the floor. } \\
\text { Contract your buttocks muscles } \\
\text { and raise your pelvis and lower } \\
\text { back. Hold the position and } \\
\text { straighten one leg. Hold that } \\
\text { position for 3-5 seconds and } \\
\text { slowly lower back. Repeat with } \\
\text { opposite leg. }\end{array}$ \\
\hline
\end{tabular}




\begin{tabular}{|c|c|c|c|c|c|}
\hline $\begin{array}{l}8 \text { - } \\
\text { Crunch w/knee lift }\end{array}$ & $\square$ & $\int_{12}^{2}$ & 48 & $3 \times 10$ & $\begin{array}{l}\text { Sit on the floor with your hands } \\
\text { behind your head. leaning } \\
\text { backward, raise your legs } \\
\text { slightly from the floor and find } \\
\text { your balance. Alternately move } \\
\text { opposite knee and elbow } \\
\text { toward each other. }\end{array}$ \\
\hline $\begin{array}{l}9- \\
\text { The plank }\end{array}$ & 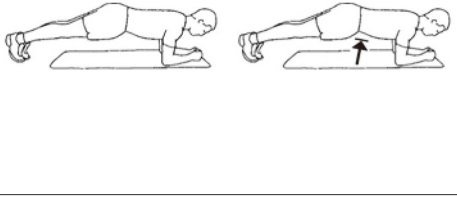 & $\int$ & & $3 \times 10$ & $\begin{array}{l}\text { Stand on your elbows and the } \\
\text { tips of your toes, find the } \\
\text { neutral position of your back } \\
\text { and pelvis. "Flatten" your } \\
\text { abdomen, holding the position } \\
\text { for } 10-15 \text { seconds. }\end{array}$ \\
\hline $\begin{array}{l}10 \text { - } \\
\text { Side-lying } \\
\text { side lift }\end{array}$ & द & 4 & 10 & $3 \times 10$, each side & $\begin{array}{l}\text { Lie on your side supporting } \\
\text { yourself on your elbow. Find the } \\
\text { neutral position of your back and } \\
\text { pelvis. "Flatten" your abdomen } \\
\text { and raise your pelvis until your } \\
\text { body is straight. Hold for } 10-15 \\
\text { seconds and rest equally. Repeat } \\
\text { to opposite side. }\end{array}$ \\
\hline
\end{tabular}

\section{Appendix 2B}

Program B

Helicopter pilots with transient low back pain

\section{Angle bench}

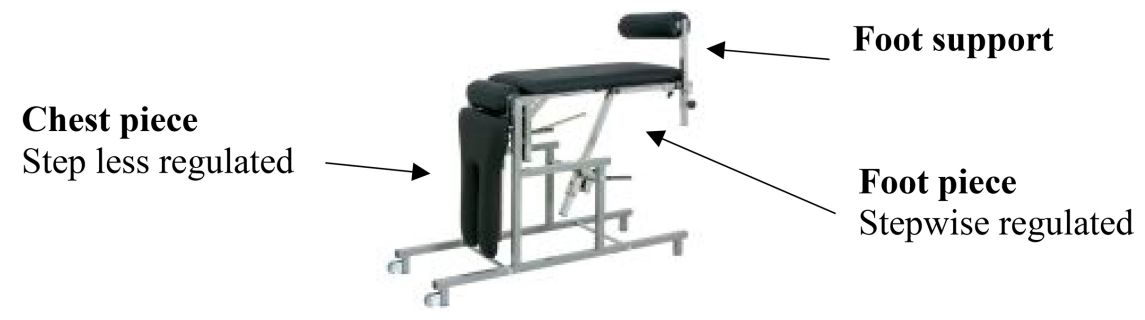

\section{General instructions}

Basic period over 36 training sessions not more frequently than every other day. 10-minute general warm up before start.

\section{Extension}

Bench settings: Foot piece; one clicks down $\left(10^{\circ}\right)$

Chest piece; $41^{\circ}$ in relation to foot piece

Execution: Lift upper torso in a steady fast tempo to a straight body line avoiding any extension, also of the cervical spine. Maintain an isometric straight body line for 6 seconds. Eccentric phase of at least 7 seconds returning to starting point. "Bracing" initiated and released between each repetition reducing the intensity of the bracing procedure with improved skill. Number of repetitions according to submaximal individual capacity in 4 series with increasing numbers according to reversed pyramid protocol of maximally 7-6-5-4 repetitions. At least 4 training sessions at any level before increasing by one repetition per series. One-minute intermission involving brisk walking or biking between every series. Once achieved 7-6-5-4 repetitions the load is increased by applying weight west starting with $2 \mathrm{~kg}$ and increasing load by $1 \mathrm{~kg}$ every fourth training session. 

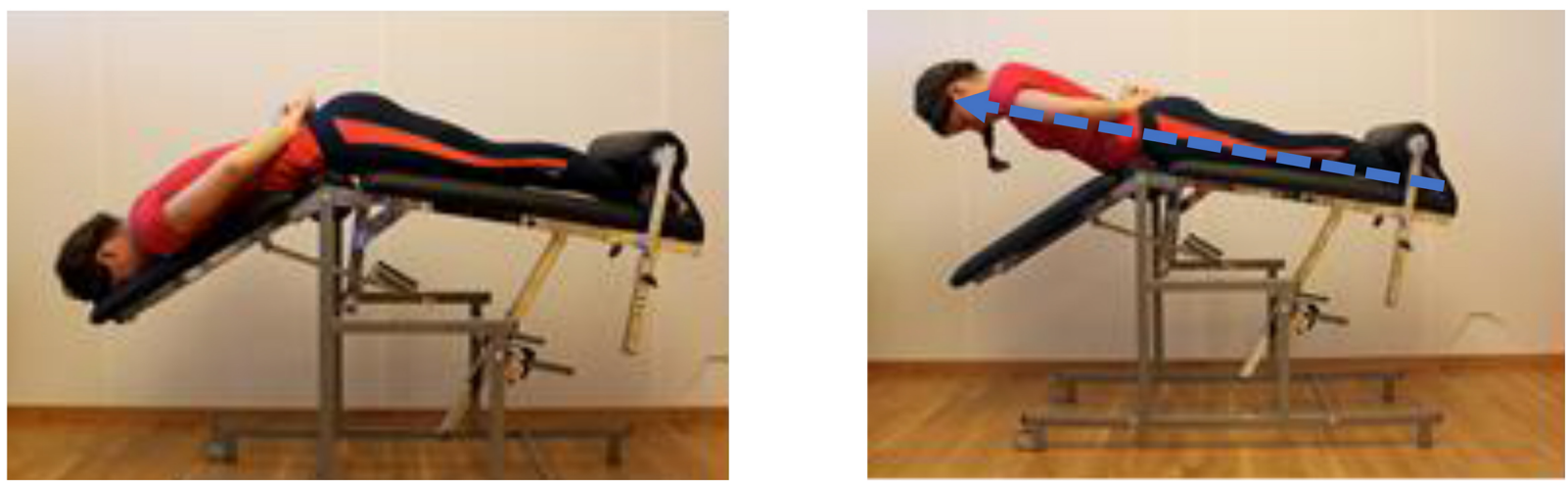

2 "Curl-up"

\section{Floor mat}

Execution: Hand placed under the lumbar spine for support. Tongue firmly against the palate. One knee bent at $90^{\circ}$ with sole of foot against the floor. Steady fast lifting the upper torso in a straight line avoiding "pulling-up" by head flexion to a low angle. Maintain an isometric position for 6 seconds. Eccentric phase of at least 7 seconds returning to starting point. "Bracing" initiated and released between each repetition reducing the intensity of the bracing procedure with improved skill. Number of repetitions according to submaximal individual capacity in 4 series with increasing numbers according to reversed pyramid protocol of maximally 7-6-5-4 repetitions. At least 4 training sessions at any level before increasing by one repetition per series. One-minute rest on floor between series. Once achieved 7-6-5-4 repetitions the load is increased by applying weight west starting with $1 \mathrm{~kg}$ and increasing load by $1 \mathrm{~kg}$ every fourth training session.
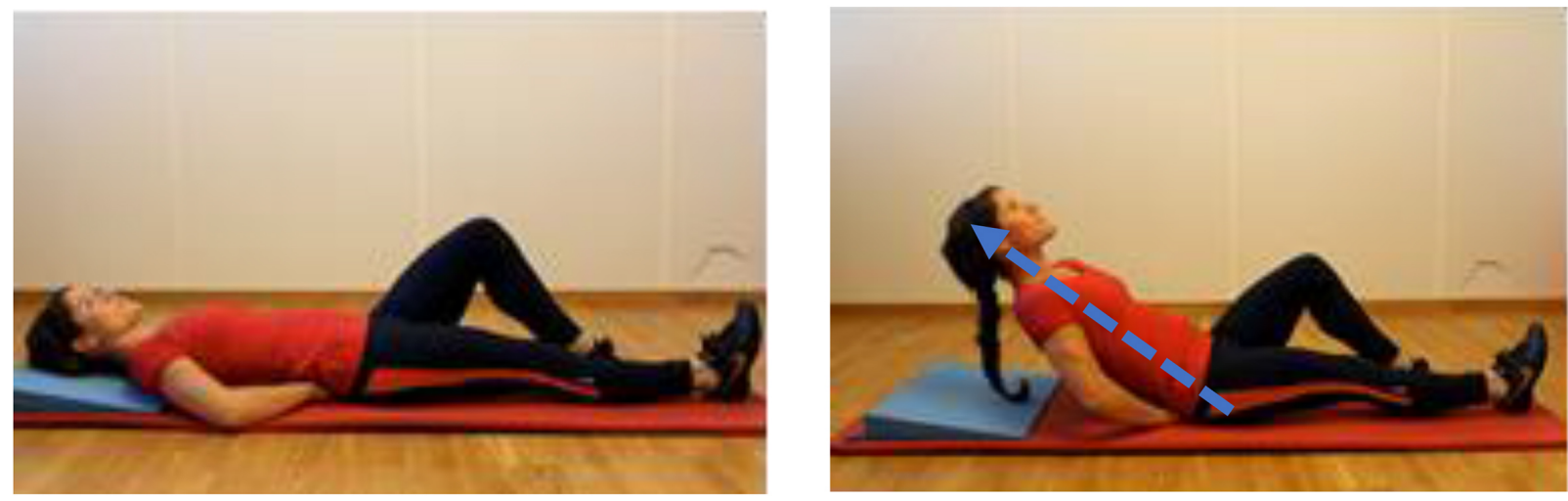

\section{Bilateral leg extensions}

Bench settings: Foot piece; one clicks down $\left(10^{\circ}\right)$

Chest piece vertical

Execution: Prone position with chin and head resting on the hands. Raise both legs simultaneously in a steady fast tempo while gradually externally rotating both feet. Keep the legs (knees) fully extended at all times. The body should be in a straight line avoiding hyperextension. The overall body position on the angle bench must always be customized according to individual capacity as to seek the best possible working angle. Maintain an isometric position for 6 seconds. Eccentric phase of at least 7 seconds returning to starting point. "Bracing" initiated and released between each repetition reducing the intensity of the bracing procedure with improved skill. Number of repetitions according to submaximal individual capacity in 4 series with increasing numbers according to reversed 
pyramid protocol of maximally 7-6-5-4 repetitions. At least 4 training sessions at any level before increasing by one repetition per series. One-minute intermission involving brisk walking or biking between every series. Once achieved 7-6-5-4 repetitions the load is increased by applying weights on both ankles starting with $1 \mathrm{~kg}$ during four sessions. Load is increased to a maximum of $2 \mathrm{~kg}$ to be continued throughout the remaining basic training program of 36 sessions.
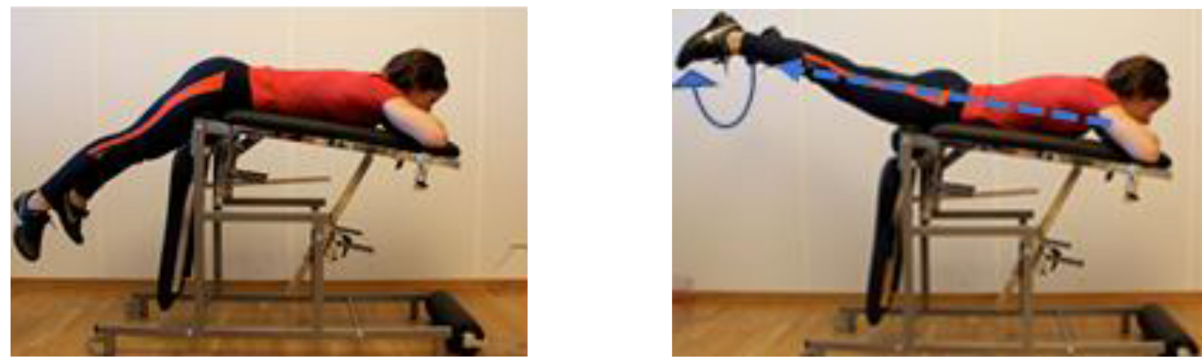

\section{Upper Torso Lateral Raise}

Bench settings: $1^{\text {st }}$ set foot piece to $25^{\circ}$ ( 3 clicks down from level position).

$2^{\text {nd }}$ adjust chest piece to appropriate angle according to individual capacity (ranging from $+5^{\circ} / 0^{\circ}\{$ level $\} /$ $\div 5^{\circ} / \div 10 / \div 15^{\circ}$ or $\div 20^{\circ}$ )

Execution: Sideways on the angle bench with lower leg bent $90^{\circ}$ and securing the lower leg with a straight upper leg beneath foot support. Body in a straight line with feet, pelvis and shoulder centrally placed on the bench. The working angle of the chest piece is set according to individual capacity. Lift the torso sideways to a straight position in a steady fast tempo avoiding any lateral flexion of the cervical spine. Maintain an isometric position for 6 seconds. Eccentric phase of at least 7 seconds returning to starting point. "Bracing" initiated and released between each repetition reducing the intensity of the bracing procedure with improved skill. Number of repetitions according to submaximal individual capacity in 4 series with increasing numbers according to reversed pyramid protocol of maximally 7-6-5-4 repetitions. The exercises are performed consecutively on every other side series by series. Increasing load by increasing working angle in increments as suggested every fourth training session. After maximum working angle is achieved, further loading by weight west starting with $2 \mathrm{~kg}$ and adding $1 \mathrm{~kg}$ every fourth training session.

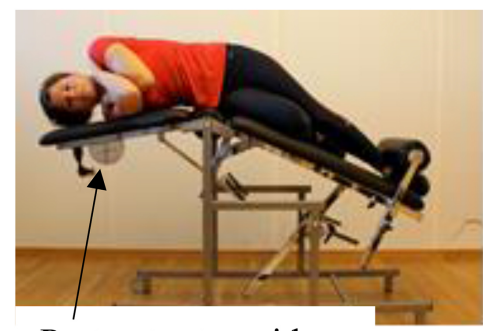

Protractor to guide the working angle

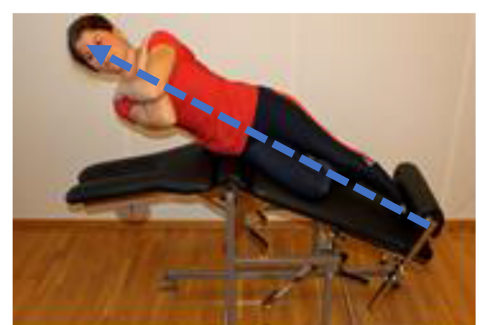

